\title{
MedienPädagogik
}

www. medienpaed.com

\section{Denn wir wissen nicht, was sie tun ... Portfolios zur Dokumentation von Kompetenzen in einem weiterbildenden Masterstudiengang}

\author{
Olaf Zawacki-Richter ${ }^{1}$, Eva Maria Bäcker² und Anke Hanft ${ }^{3}$
}

\begin{abstract}
Im vorliegenden Beitrag wird am Beispiel eines internetgestützten, weiterbildenden Studiengangs gezeigt, wie die Portfoliomethode als kompetenzbasierte Prüfungsleistung in ein Blended Learning Design integriert werden kann. Im Rahmen einer qualitativen Analyse von Projektportfolios wird untersucht, wie Studierende über ihren Kompetenzentwicklungsprozess reflektieren und welche Kompetenzen auf diese Weise dokumentiert werden.
\end{abstract}

\section{Einführung}

Der im Bolognaprozess verankerte Paradigmenwechsel von der Inputorientierung (was soll vermittelt werden?) zur Outcomesorientierung (was sollen die Studierenden können?) erfordert die Ausrichtung der Prüfungsleistungen an die zu erwerbenden Kompetenzen. Während die Umstellung der Studienorganisation weitgehend abgeschlossen ist, kann das kompetenzbasierte Assessment als «ungelöste Hausaufgabe» gelten (Stratmann, Preussler \& Kerres, 2009). Reinmann (2007) spricht gar von einer «Kompetenzlüge» (S. 13). Die Gegenwärtige Prüfungspraxis besteht in der Regel aus einem universitären Dreikampf: Klausuren, Referate, Hausarbeiten.

Ein Grund für die mangelnde Umsetzung kompetenzbasierter Ansätze ist sicherlich die methodische Schwierigkeit der Messung von Kompetenzen (vgl. Erpenbeck \& v. Rostenstiel, 2003).

Weinert (2001) beschreibt Kompetenzen als die bei Individuen verfügbaren oder durch sie erlernbaren kognitiven Fähigkeiten und Fertigkeiten, um bestimmte Probleme zu lösen, sowie die damit verbundenen motivationalen, volitionalen (absichtsbezogenen) und sozialen Bereitschaften und Fähigkeiten, um die Problemlösungen in variablen Situationen erfolgreich und verantwortungsvoll umsetzen

1 Dr. Olaf Zawacki-Richter, FernUniversität in Hagen, Vertretungsprofessor für Bildungstechnologie und Lehrbeauftragter im MBA Bildungsmanagement der Universität Oldenburg, olaf.zawacki-richter@fernuni-hagen.de

2 Dr. Eva Maria Bäcker, FernUniversität in Hagen, Wissenschaftliche Mitarbeiterin im Lehrgebiet Bildungstechnologie, eva.baecker@fernuni-hagen.de

3 Prof. Dr. Anke Hanft, Carl von Ossietzky Universität Oldenburg, Sprecherin des Arbeitsbereichs Weiterbildung und Bildungsmanagement, Wissenschaftliche Leiterin des MBA Bildungsmanagement, anke.hanft@uni-oldenburg.de 
zu können. Die Verzahnung von Kognition und Motivation ist ein Wesensmerkmal kompetenten Handelns (Weinert, 1996). Dies findet seinen Ausdruck im selbstgesteuerten Lernen als Mechanismus der Kompetenzentwicklung (vgl. stellvertretend Knowles, 1975). Das schliesst ein, dass selbständig Ziele gesetzt, Pläne und Strategien zu ihrer Verwirklichung erarbeitet und erprobt werden und aus dabei entstehenden Erfahrungen gelernt wird. Entsprechend definieren Erpenbeck \& von Rosenstiel (2003) Kompetenzen auch als Selbstorganisationsdispositionen. Kompetenz setzt sich also aus Wissen, Erfahrungen und Fähigkeiten zur Anwendung und Umsetzung des Wissens, der Kenntnisse, Fähigkeiten und Fertigkeiten zusammen (Zawacki-Richter, Hasebrook \& Muckel, 2009). Connell, Sheridan \& Gardner (2003) beschreiben Kompetenzen auch trefflich als «realized abilities». Diese Performanz in neuen, unsicheren Kontexten kann dokumentiert werden, um Kompetenzen sichtbar und somit auch bewertbar zu machen. Hier liegt auch der wesentliche Unterschied zwischen Qualifikation und Kompetenz. Eine Qualifikation im traditionellen Sinne dokumentiert nichts weiter als die Performanz in einer künstlichen Situation, einer Prüfung, in der Wissen abgefragt aber nicht kompetentes Handeln gezeigt wird.

Eine hierfür geeignete Methode ist das Portfolio - oder E-Portfolio für die elektronische Form: «Portfolios [...] are always a goal driven, organized collection of items (artifacts) that demonstrate a learner's expansion of knowledge and skills over time» (Walti, 2004, S. 157).

In den weiterbildenden, internetbasierten Studiengängen an der Universität Oldenburg, sind Lernportfolios als Prüfungsleistung in den Prüfungsordnungen verankert. In diesem Aufsatz soll zunächst am Beispiel des Studiengangs MBA Bildungsmanagement die didaktische Einbettung der Portfolios im Studien- und Modulverlauf dargestellt werden. In einem zweiten Schritt sollen im Rahmen einer qualitativen Textanalyse Portfolios aus verschiedenen Modulen des Studiengangs ausgewertet werden. Dabei untersuchen und beschreiben wir entsprechend dem Kodierparadigma der Grounded Theory wie und vor allem über welche Kompetenzen die Studierenden in den Portfolios reflektieren. Als ersten Zugang zum empirischen Material legen wir als Theoriefolie das Kompetenzmodell von Erpenbeck \& Heyse (1999) zugrunde, das Kompetenzen in vier Klassen einteilt: personale Kompetenzen, aktivitäts- und handlungsorientierte Kompetenzen, sozial-kommunikative und fachlich-methodische Kompetenzen. Der darauf aufbauende «Kompetenzatlas» (Heyse \& Erpenbeck, 2004) dient uns als Orientierung.

Wir wollen zeigen, wie die Portfoliomethode didaktisch in Studiengängen integriert und verbindlich als Prüfungsleistung verankert werden kann. Anhand des empirischen Materials soll gezeigt werden, wie die Studierenden über ihre Kompetenzentwicklung reflektieren und welche Kompetenzen auf diese Weise dokumentiert werden. 


\section{Integration von Portfolios in internetbasierten Studiengängen am Beispiel der Universität Oldenburg}

Die Universität Oldenburg nimmt eine Pionierrolle bei der Entwicklung von berufsbegleitenden und/oder weiterbildenden Studiengängen ein. Bereits im Sommersemester 2003 wurde der erste internetbasierte, weiterbildende Bachelorstudiengang in Business Administration eingeführt. Schon 1999 entwickelte die Universität gemeinsam mit dem University of Maryland University College (USA) einen OnlineMaster of Distance Education Studiengang. Gegenwärtig werden die folgenden weiterbildenden Studiengänge angeboten:

- Bachelorstudiengang «Business Administration» (BA)

- Bachelorstudiengang «BWL für Spitzensportler/innen» (BA)

- Masterstudiengang «Bildungsmanagement» (MBA)

- Masterstudiengang «Informationsrecht» (LL.M.)

- Masterstudiengang «Distance Education» (MDE) in Kooperation mit dem University of Maryland University College (USA)

- Masterstudiengang «Renewable Energy» (MSc)

- Masterstudiengang Innovationsmanagement (MA)

Das didaktische Design der oben genannten Studiengänge ist auf die Bedürfnisse und Anforderungen der berufstätigen Zielgruppen zugeschnitten. Im Mittelpunkt steht ein anwendungsbezogenes, aktives Lernen an realen Projekten, die die Teilnehmer/innen aus ihrem eigenen Arbeitskontext einbringen und in Teams bearbeiten. Dabei wird in sechs Studiengängen der Ansatz des Blended Learning verfolgt ${ }^{4}$, um eine maximale räumliche und zeitliche Flexibilität des Studienangebots bei gleichzeitiger direkter Interaktion zwischen Lernenden und Lehrenden sowie zwischen den Lernenden untereinander (Knüpfung sozialer Kontakte, Gruppenbildung, Themenfindung, Ergebnispräsentation und -diskussion) zu ermöglichen (vgl. Sauter \& Sauter, 2002). In Abbildung 1 ist die Blended Learning Struktur eines Studienmoduls dargestellt.

4 Der Master of Distance Education ist als reiner Online-Studiengang ohne Präsenzphasen konzipiert. 


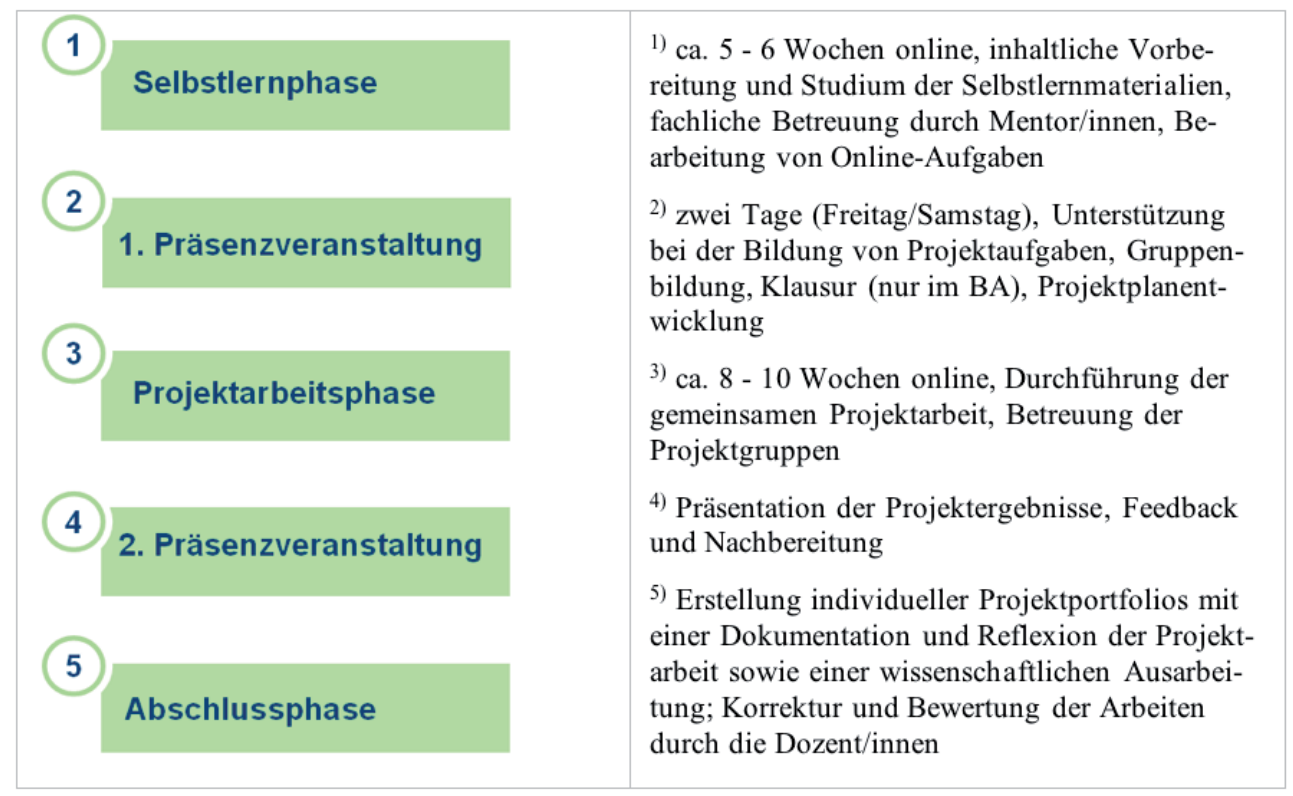

Abb. 1: Modulablauf in den berufsbegleitenden Studiengängen der UOL

Die folgenden Ausführungen konzentrieren sich auf den MBA Studiengang Bildungsmanagement. In den Mittelpunkt des Interesses rückt das Projektportfolio, das zum Abschluss eines Studienmoduls (Nachbereitungsphase) von allen Studierenden als Einzelleistung erbracht wird. Nach der Systematik von Baumgartner, Himpsl \& Zauchner (2009) handelt es sich hierbei um ein individuelles Personenportfolio, bei dem es sowohl um die produktorientierte (summativ) als auch prozessorientierte (formativ) Reflektion des Lernprozesses geht.

In der Handreichung für Studierende und Lehrende im MBA Bildungsmanagement wird die Funktion des Portfolios wie folgt erläutert: «Das Portfolio ist eine zielgerichtete Sammlung von Materialien und Dokumenten, die einen Leistungsbereich im Zeitverlauf dokumentieren soll. Das Portfolio ist somit auch Bestandteil der Selbstevaluation und gibt den Teilnehmern/-innen und Aussenstehenden einen Überblick über persönlichen Lernerfolg, Ziele und Ergebnisse der Projekte». In der Handreichung finden die Studierenden auch Hinweise zur Strukturierung der Portfolioarbeit.

Das Projektportfolio knüpft an den Verlauf der Projektphase nach der Ergebnispräsentation an und besteht aus zwei Teilen:

- Einer Darstellung der Projektarbeit und des eigenen Kompetenzzuwachses und

- einer wissenschaftlichen Ausarbeitung zu einer zentralen Fragestellung des Projektes. 
Das Projektportfolio («Lernportfolio») ist als verbindliche Prüfungsleistung in der Prüfungsordnung des Studiengangs verankert und wird bei der Festlegung der Modulnote deutlich gewichtet: "Die Bewertung der Ergebnispräsentation geht zu einem Drittel und die des Lernportfolios zur Bearbeitung der Projektaufgaben zu zwei Dritteln in die Fachnote des jeweiligen Studienmoduls ein» (§13, Satz 9 Prüfungsordnung MBA). Die Dozenten/-innen erhalten ein Bewertungsschema für die Ergebnispräsenation und das Lernportfolio, an dem sie sich orientieren können. Die Teilnehmer/innen im MBA Bildungsmanagement sind im Durchschnitt ca. 37 Jahre alt. Nahezu 100\% sind berufstätig und besetzen Leitungspositionen (Kanzlerin, Geschäftsführer, Dezernatsleiter) oder Positionen des mittleren Managements (Fachbereichsleiter, Fakultätsgeschäftsführer, Programm-Manager) in Bildungsund Wissenschaftsinstitutionen. 30 bis $40 \%$ der Teilnehmer/innen kommen aus der öffentlich finanzierten oder privatwirtschaftlichen Weiterbildung. Etwa 10\% sind in der Bildungspolitik bzw. -administration tätig (z. B. in Ministerien oder Akkreditierungsagenturen). Alle Studierende im MBA haben bereits einen ersten Studienabschluss (60\% mit Universitätsabschluss, 25\% mit Fachhochschulabschluss, 10\% sind promoviert).

\section{Methodische Vorgehensweise}

\subsection{Das Kompetenzmodell nach Erpenbeck et al.}

Im Folgenden sollen nun die wichtigsten theoretischen Bezüge dieser Untersuchung erläutert werden. Weinert (2001) beschreibt Kompetenzen als die bei Individuen verfügbaren oder durch sie erlernbaren kognitiven Fähigkeiten und Fertigkeiten, um bestimmte Probleme zu lösen, sowie die damit verbundenen motivationalen, volitionalen (absichtsbezogenen) und sozialen Bereitschaften und Fähigkeiten, um die Problemlösungen in variablen Situationen erfolgreich und verantwortungsvoll umsetzen zu können. Kompetenz setzt sich also aus Wissen, Erfahrungen und Fähigkeiten zur Anwendung und Umsetzung des Wissens, der Kenntnisse, Fähigkeiten und Fertigkeiten zusammen. Connell, Sheridan \& Gardner (2003) beschreiben Kompetenzen auch als «realized abilities».

Die Verzahnung von Kognition und Motivation ist ein Wesensmerkmal kompetenten Handelns (Weinert, 1996). Dies findet seinen Ausdruck im selbstgesteuerten Lernen als Mechanismus der Kompetenzentwicklung (vgl. stellvertretend Knowles, 1975). Das schliesst ein, dass selbständig Ziele gesetzt, Pläne und Strategien zu ihrer Verwirklichung erarbeitet und erprobt werden und aus dabei entstehenden Erfahrungen gelernt wird. Entsprechend definieren Erpenbeck \& von Rosenstiel (2003) Kompetenzen auch als Selbstorganisationsdispositionen. 


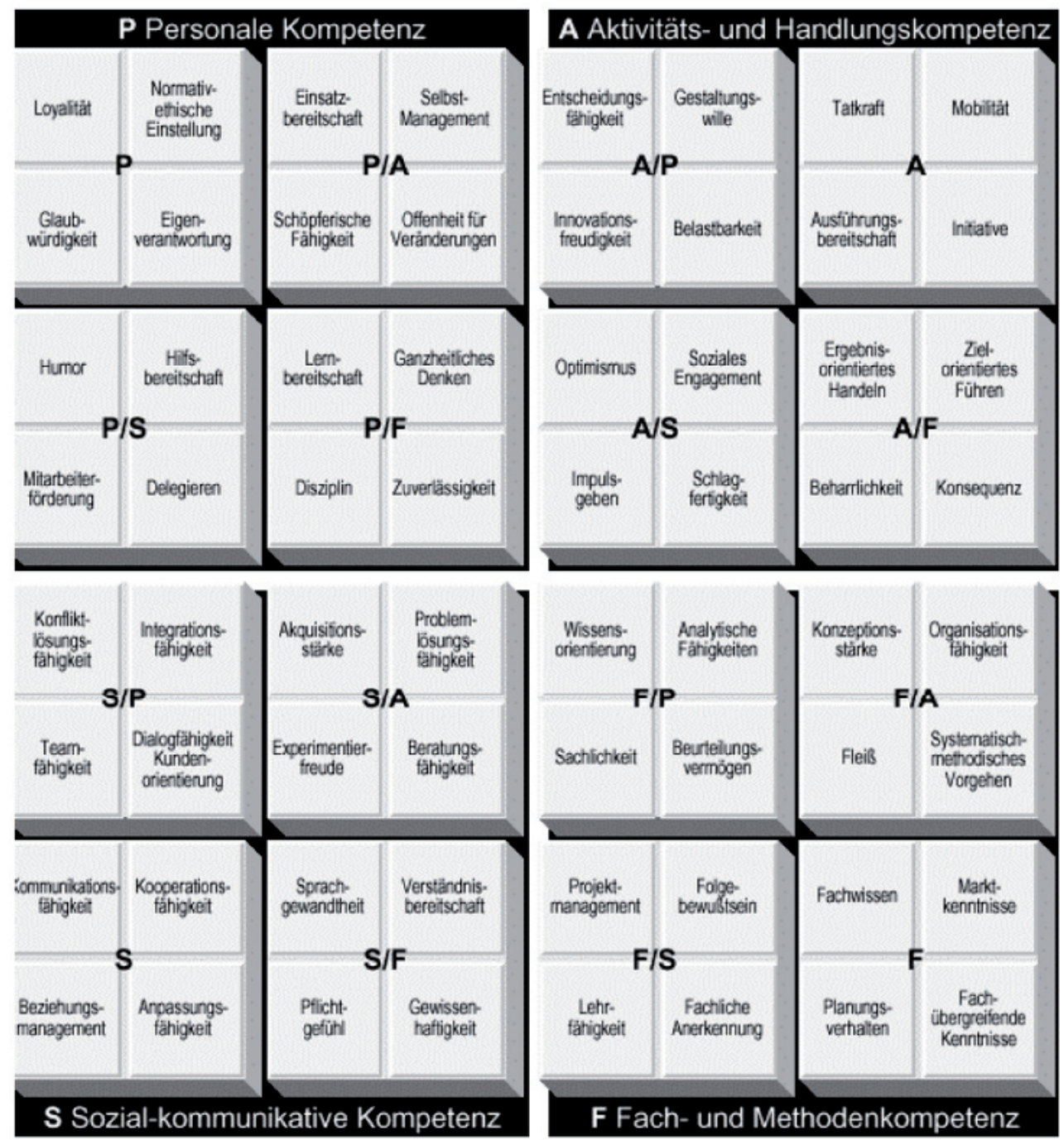

Abb. 2: Der Kompetenzatlas (Heyse \& Erpenbeck, 2004, S. XXI)

Im Hinblick auf die Systematik und Taxonomie von individuellen Kompetenzen wurde von Erpenbeck und Kollegen (Erpenbeck \& Heyse, 1999; Erpenbeck \& v. Rosenstiel, 2003; Heyse \& Erpenbeck, 2004) ein Kompetenzmodell vorgelegt, mit dem sich die unterschiedlichen Kompetenzdimensionen operationalisieren lassen, um damit ein «wenig Zeit beanspruchendes Instrumentarium zur Messung von Kompetenzen und Kompetenzentwicklungen» (Erpenbeck \& Heyse, 2007, S. 24) zu entwickeln. Kompetenzen werden in Kompetenztypen, -klassen und-gruppen eingeteilt und in einem «Kompetenzatlas» zusammengefasst (siehe Abbildung 2). 
Die vier Kompetenzklassen umfassen:

1. personale Kompetenzen (Fähigkeit, reflexiv und selbstorganisiert zu handeln, sich selbst einschätzen zu können, Zuverlässigkeit, Lernbereitschaft),

2. aktivitäts- und umsetzungsorientierte Kompetenzen (z. B. Flexibilität, Selbständigkeit, Kreativität, Initiative),

3. fachlich-methodische Kompetenzen (z. B. Sachlichkeit, Projektmanagement),

4. sozial-kommunikative Kompetenzen (z. B. Teamfähigkeit, Kritikfähigkeit).

Querschnittskompetenzen sind komplexe Kompetenzen, deren Facetten in alle vier Kompetenzklassen hineinspielen. Beispiele für Querschnittskompetenzen sind Medienkompetenz, Management- oder Führungskompetenz, Innovationskompetenz und interkulturelle Kompetenz (vgl. Zawacki-Richter \& Hasebrook, 2005). Querschnittskompetenzen werden auch als Metakompetenzen bezeichnet (Weinert, 2001).

\subsection{Qualitative Analyse von Kompetenzen in Portfolios}

Vor dem beschriebenen Hintergrund des Kompetenzbegriffs sind Kompetenzen von Studierenden nicht wie erworbene Fach- und Methodenkenntnisse direkt überprüfbar, sondern nur aus der Anwendung von konkreten Fähigkeiten in Handlungssituationen bewertbar (Performanz). Ausgehend von der Fragestellung, wie Studierende ihre Kompetenzentwicklungsprozesse im Portfolio reflektieren und welche Kompetenzen für die Lehrenden auf diese Weise dokumentiert werden, soll in der vorliegenden Untersuchung analysiert werden, ob die Portfoliomethode als kompetenzbasierte Prüfungsleistung geeignet ist, um anhand der Reflexionen der Studierenden über ihren Lernprozess, ihre Kompetenzen sichtbar zu machen. Als Datengrundlage dient der erste Teil der schriftlichen Projektportfolios des MBA Studiengangs Bildungsmanagement. Diese Prüfungsleistung schliesst sich an die Ergebnispräsentation der Projektphase an und beinhaltet eine Darstellung der Projektarbeit sowie des eigenen Kompetenzzuwachses aus der (subjektiven) Perspektive der Studierenden. Ausgehend von den vier Kompetenzklassifizierungen (Personale Kompetenz, Aktivitäts- und Handlungskompetenz, Sozialkommunikative Kompetenz, Fach- und Methodenkompetenz) von Heyse \& Erpenbeck soll der Kompetenzatlas als Theoriefolie über die schriftlichen Reflexionen der Kursteilnehmer/innen gelegt werden. Unter Anwendung der Grounded Theory wurden die Protokolle der Studierenden kodiert ${ }^{5}$, um die Reflexionen als theoretische Konstrukte den Kompetenzfeldern im Kompetenzmodell zuordnen zu können.

5 Mit dem Prozess des «Kodierens» ist die gedankliche und sprachliche Aktivität verstanden, aus den empirischen Materialien einzelfallübergreifende, verallgemeinernde Begriffe zu bilden. Das Wesentliche soll herausgearbeitet und zusammengefasst werden und kann dann zum Vergleich bestehender Konzepte verwendet werden. 
Die Grounded Theory stellt eine interpretative Methode dar, die eine «systematische Reihe von Verfahren benutzt, um eine induktiv abgeleitete gegenstandsverankerte Theorie über ein Phänomen zu entwickeln» (Strauss \& Corbin, 1996, S. 8f.).

In ihrer Alltagswelt werden von den Akteuren unbewusst Gesprächsinformationen selektiert und während eines Gesprächs das Gegenüber in «Schubladen» eingeordnet. Wahrgenommene Eigenschaften werden verallgemeinert oder auch stigmatisiert. Wahrgenommene Verhaltensweisen einer Person - methodisch als Indikatoren bekannt - werden in einer typisierenden Weise zusammengefasst. Mittels der Kodierung der Grounded Theory werden Konstrukte, theoretische Begriffe, in Zusammenhang mit Indikatoren gebildet, was auf dem sogenannten KonzeptIndikator-Modell basiert (vgl. Brüsemeister 1999: 137 ff.; Strauss/Corbin 1996: 43 ff.; Schnell u. a. 1992: $37 \mathrm{ff}$.$) .$

Strauss und Corbin (1996) betonen die Wichtigkeit der durchdachten Fragestellung beim Umgang mit den Daten sowie die Notwendigkeit eines Vergleichs der Konzepte im Hinblick auf die «theoretische Sensibilität»: «Kodieren stellt die Vorgehensweise dar, durch die die Daten aufgebrochen, konzeptualisiert und auf neue Art zusammengesetzt werden» (Strauss \& Corbin, 1996, S. 39). Dies beschreibt den zentralen Prozess, durch den aus den Daten Theorien entwickelt werden.

Das Entscheidende beim Kodieren ist das Auffinden von Begriffen (Konzepten, Konstrukten, Kategorien) und keine Paraphrasierung der Daten. Die wesentliche Inhalte der Portfolios werden somit konkret auf einem abstrakteren Niveau gebündelt.

Die Kodierschritte bauen aufeinander auf: Das offene Kodieren zeichnet sich dadurch aus, dass das Datenmaterial Zeile für Zeile durchgegangen und mit einem Kode als Etikett versehen wird. Phänomenbeschreibungen werden mit potentiell passenden abstrakten Begriffen gekennzeichnet. Vergleichbar mit einem Brainstorming beinhaltet diese Kodierungsform Möglichkeiten zu Interpretationen und verschiedenen Lesarten der Daten. Die Intention ist die Bildung von gegenstandsbezogenen, typisierenden und abstrakten Begriffen, um die Daten mehrerer Fälle vergleichbar zu machen.

Unabdingbar ist demnach, dass permanent Fragen an die Daten gestellt und alle Ereignisse hinsichtlich Ähnlichkeiten und Unterschieden miteinander verglichen und zu Kategorien gruppiert werden (Strauss \& Corbin, 1996, S. 54f.).

\subsection{Qualitatives Sampling und Thematisches Kodieren}

Die Stichprobe dieser Untersuchung umfasst 23 Portfolios aus den Modulen «Ansätze internetgestützten Lernens» und "Didaktik des E-Learning» aus dem MBA Studiengang Bildungsmanagement. Für die Auswahl der Stichprobe für die komparative Analyse wurden im Prozess des offenen Kodierens alle Portfolios kodiert und später in Gruppen von Gegenbeispielen im Sinne einer kontrastierenden Fallauswahl eingeteilt. Zur Überprüfung, ob sich in den schriftlichen Reflexionen die vier 
Kompetenztypenklassen des Heyse \& Erpenbeck-Modells widerspiegeln, erfolgt zuletzt eine Auswahl derjenigen Portfolios, die zueinander maximal kontrastieren. Somit orientiert sich das Sampling an den Kategorien von Heyse \& Erpenbeck, um später bei der Analyse einer systematischen Untersuchung standhalten zu können, inwieweit sich das Modell von Heyse \& Erpenbeck auf die Portfolios anwenden lässt. Die ermittelten kontrastierenden Portfolios durchliefen einen nochmaligen Kodierschritt des offenen Kodierens, welcher unter Verwendung des computergestützten Analyse-Software-Programms MAX-QDA vorgenommen wurde. Während des Kodierprozesses erfolgte ein fortwährender Vergleich der mittlerweile auf vier kontrastierende Portfolios reduzierten Daten. Durch Angleichung der in den Codes aus dem ersten Kodierschritt der Vollerhebung übereinstimmender Situationsbeschreibungen konnte eine überprüfbare Datenkontrolle gewährleisten werden.

Im nächsten Analyseschritt war eine Modifizierung des Pfads des klassischen Kodierprozesses der Grounded Theory notwendig, um die 64 Kompetenzgruppen des Heyse \& Erpenbeck Modells auf die gewonnenen Kategorien aus den Portfolios anwenden zu können und damit gleichzeitig die Vergleichbarkeit der Daten zu erhöhen.

Nach dem Prozess des zweiten Durchgangs des offenen Kodierens wurden die 64 Kompetenzen aus dem Kompetenzatlas als vordefiniertes Kategoriensystem in MAX-QDA eingegeben. Dieses Vorgehen, auch als thematisches Kodieren benannt (Flick, 1996, S. 206 f.; Kuckartz, 2007, S. 84), eignet sich für vergleichende Daten mit vorab festgelegten Kategorien, wie es bei den definierten Kompetenzen von Heyse \& Erpenbeck der Fall ist. Hierbei werden die festgelegten Kategorien, hier die Kompetenzen, als thematische Bereiche auf den einzelnen Fall abgeglichen. Die Interpretation der Daten geht von einem theoretischen «Konzept der Verteilung der Perspektiven auf einen bestimmten Gegenstand oder Prozess» (ebd., S. 210 f.) aus.

Das Ergebnis ist eine thematische Kompetenz-Struktur eines jeden Portfolios, die eine eindeutige Einordnung zu einer Kompetenzgruppe von Heyse \& Erpenbeck sichtbar werden lässt. Diese Einordnung dient weiter zur Identifizierung von Gemeinsamkeiten und Kontrasten der von den Studierenden reflektierten Kompetenzprozessen. Somit lässt sich die Gültigkeit des Kompetenzatlas von Heyse \& Erpenbeck fallbezogen auf die Reflexionen in den Portfolios überprüfen. Wir wollen damit zeigen, mit welcher Reichweite sich das Kompetenzmodell auf Studienportfolios übertragen werden lässt und damit eine didaktische Verankerung der Portfoliomethode in Studiengängen legitimiert. 


\section{Diskussion der Ergebnisse}

Die durch die offene Kodierung ermittelten Kategorien konnten eindeutig den vier Kompetenzklassen von Heyse \& Erpenbeck zugeordnet werden. In den Portfolios wurden Handlungen der Studierenden sichtbar gemacht, Strategien aufgespürt und Einstellungen aufgedeckt. Durch die thematische Kodierung konnten die Portfolios in sogenannte «Kompetenzlandkarten» der Studierenden umgewandelt werden.

Strauss \& Corbin (1996) beschreiben den Weg, Prozesse zu untersuchen, «indem man Schnappschüsse von Handlungen/Interaktionen macht und diese zu einer Sequenz oder Serie verknüpft» (S. 119). Mit Hilfe des Kodierparadigmas kann ein zu analysierendes Phänomen (hier: die Kompetenzen der Studierenden) beschrieben werden. So können systematisch in den Daten komplexe Beziehungen herausgearbeitet und verglichen werden. Konkret geht es in dieser Untersuchung darum, die Kompetenzen, die dazu führen, individuelle Studienziele mit der geforderten Gruppenarbeit und der anschliessenden Präsentation des Ergebnisses in der Gruppe zu vereinbaren, in den Texten durch Verwendung des Kompetenzrasters sichtbar zu machen. Um den Komplex der Kompetenzen, die bei einer OnlineGruppenarbeit notwendig sind, vergleichen zu können, wurden aus den Portfolios die im ersten offenen Kodierdurchgang aufgefundenen Kategorien in Gegenüberstellung mit den Kodes der 64 Kompetenzen der thematischen Kodierung daraufhin überprüft, inwiefern sie sich in den einzelnen Kompetenztypen unterscheiden. Als Beispiel: Bei Vermutungen für eine vorliegende Kompetenz (z. B. personale Kompetenz) wird im Text nach den Bedingungen für das Auftreten der Ausprägung dieser Kompetenz in einem Projektbereich (z. B. Themenwahl innerhalb der Projektgruppe) gesucht. Welche Handlungen sind erkennbar (hier: Positionierung des eigenen Themas ohne Berücksichtigung der Wünsche der Gruppe)? Welche Konsequenzen ergeben sich durch diese Strategie für den Akteur (hier: Durchsetzung der beruflichen eigenen Ziele; «Leaderposition» in der Gruppe)? Als analytische Geschichte könnte in diesem Fall resümiert werden: «Besitzt der Kompetenztyp «personale Kompetenz) die Möglichkeit zur Durchsetzung seiner persönlich gesteckten Ziele, dann wird er diese Ziele unbeirrt auch in einer Gruppe verfolgen und die Gruppe als «Mittel zum Zweck〉 für seine Ziele nutzen».

Anhand der nun aufgeführten Kategorien werden die in der Analyse aus den Portfolios herausgearbeiteten Kompetenzen innerhalb der einzelnen Kompetenztypen verglichen:

- Vorbereitung auf das Online-Modul (Pre-Projekt)

- Fokus und Ziele der Weiterbildung

- Einstellung zur Online-Projektarbeit

- Problemlösungsfähigkeit

- Handlungsantrieb 
- Rolle und Position in der Onlinegruppe

- Online-Präsentation des Gruppenergebnisses

- Nachbereitung des Online-Moduls (Post-Projekt)

\section{Typ-S - Sozialkommunikative Kompetenz}

Die Präsentation der Ergebnisse beginnt mit dem Kompetenztyp «sozialkommunikativ», im folgenden Typ-S genannt. Soziale Kompetenz wird laut Erpenbeck \& von Rosenstiel (2003) als die Dispositionen benannt, «kommunikativ und kooperativ selbstorganisiert zu handeln, d. h. sich mit anderen kreativ auseinander- und zusammenzusetzen, sich gruppen- und beziehungsorientiert zu verhalten, und neue Pläne, Aufgaben und Ziele zu entwickeln» (ebd., 2003, S. XVI ).

Die Kodeverteilungen hinsichtlich der identifizierten Kompetenzen treten bei Typ$S$ (weiblich), folgendermassen auf:

\begin{tabular}{|c|c|}
\hline Persönliche KompetenzlGanzheitliches Denken / Akzent Fachkompetenz & 1 \\
\hline Sozialkommunikative KompetenzlVerständnisbereitschaft / Akzent Fachkompetenz & 1 \\
\hline Sozialkommunikative Kompetenz\Teamfähigkeit / Akzent Personale Kompetenz & 6 \\
\hline Sozialkommunikative Kompetenz\Pflichtgefühl / Akzent Fachkompetenz & 1 \\
\hline Sozialkommunikative KompetenzlKooperationsfähigkeit / Akzent Sozialkompetenz & 3 \\
\hline Sozialkommunikative KompetenzIntegrationsfähigkeit / Akzent Personale Kompetenz & 1 \\
\hline Sozialkommunikative KompetenzlBeziehungsmanagement / Akzent Sozialkompetenz & 2 \\
\hline Sozialkommunikative Kompetenz\Anpassungsfähigkeit / Akzent Sozialkompetenz & 3 \\
\hline Fach- und MethodenkompetenzlSyst.-method. Vorgehen / Akzent Aktivitätskompetenz & 3 \\
\hline Fach- und Methodenkompetenz \Projektmanagement / Akzent Sozialkompetenz & 1 \\
\hline Fach- und Methodenkompetenz \Folgebewusstsein / Akzent Sozialkompetenz & 1 \\
\hline Fach- und Methodenkompetenz \Fachwissen / Akzent Fachkompetenz & 1 \\
\hline
\end{tabular}

Abb. 3: Kodeverteilungen Typ-S aus dem Code-Matrix-Browser von MAX-QDA

Der Abbildung oben ist zu entnehmen, dass die sozialkommunikative Kompetenz mit insgesamt 17 Zuordnungen dieser Kompetenzgruppe quantitativ überwiegt. Natürlich wird nicht erwartet, dass Typ-S ausschliesslich aus sozialkommunikativen Kompetenzen besteht, denn das würde eine einseitige und für ein Studium unbrauchbare Kompetenzverteilung demonstrieren. Um erfolgreich studieren zu können, benötigt die Studentin ebenso Fach- und Methodenkompetenzen, die sich auch in ihrem Portfolio zeigen. Ihre Fach- und Methodenkompetenz ist zudem als Mischform sozialkommunikativ geprägt.

Die Studentin, die dem Typ-S zugeordnet wird, beginnt ihr Portfolio mit der Beschreibung der Aufnahme der Präsenzphase. Ihr Portfolio zeigt deutlich, dass ihr Blick auf das Modul beim Ablauf des Gruppenprozesses liegt, was sich zum einen 
in der verwendeten Sprache, wie auch in der formalen Gestaltung der Reflexion äussert. Das erste Kapitel wird mit «Reflexion der Gruppenphase» betitelt, das zweite mit «Findung des Themas für die Projektarbeit».

Das fachliche Ergebnis der Projektarbeit ist aus ihrer Perspektive ausschliesslich «Weg zum Ziel» und ist dem Prozess zweitrangig angestellt. Ihre Einstellung zur Onlinearbeit äussert sich dahingehend, dass sie den Gruppenprozess und nicht die Bearbeitung des fachlichen Themas als Herausforderung bewertet. Erfahrungen in der Projektphase zielen auf Erfahrungen in der Gruppe hin. Der Arbeitsauftrag an die Gruppe lautet, Methoden und Modelle des E-Learning zu untersuchen. Bei der Themenwahl hebt die Typ-S Studentin ihre Affinität zur Gruppenarbeit hervor, die sie auch als «passives» Mitglied einer Gruppe erkennen lässt (S\Teamfähigkeit). Sie beschreibt den Prozess der Themenfindung als «zur Zufriedenheit aller auf strategische Aspekte zur Einführung von E-Learning an Hochschulen» gelungene «effektive und sinnvoll gestaltete Projektphase» (S\Kooperationsfähigkeit).

Die Position von Typ-S in der Gruppe wird als eher unauffälliges Gruppenmitglied besetzt. Die Studentin verhält sich sehr kooperativ, bringt sich dennoch wenig in den Gruppenprozess ein. Strategien zur Bearbeitung des Moduls sucht sie nicht in der Gruppe, sondern «mittels eines Projektplanes» (F\Syst.-method. Vorgehen). Eine individuelle Problemlösungsstrategie ist nicht zu erkennen. Sie ergreift bei auftretenden Problemen nicht die Initiative, sondern verhält sich abwartend passiv. Bei einem Verständigungsproblem innerhalb der Gruppe beklagt sie, dass ihr «an dieser Stelle persönlich vermutlich die Durchführung einer Telefonkonferenz hätte helfen können». Damit offenbart sie wenig personale Kompetenz mit einem Verhalten, bei dem sie für ihre Probleme einsteht, sondern zeigt ein Verhalten, das die Reaktionen der Gruppe abwartet.

Anfangs hat Typ-S «Berührungsängste» mit dem «neuen Tool». Später wird durch die gemeinsame Verwendung der Gruppe dieses Tool zu einem «geschätzten Instrument» (S\Anpassungsfähigkeit). Die Gruppe wird somit zum «Schutzraum», den Kurs nicht allein durchlaufen zu müssen.

Bei der Präsentation der Ergebnisse ist dem Typ-S die Anerkennung (als Fremdbild) der gesamten Gruppe durch die anderen Gruppen im Modul wichtig. Hier liegen eindeutig das Gruppenergebnis und die demokratische Vorstellung der Gruppe im Vordergrund. Das Ergebnis der Gruppenarbeit wird wieder hinten angestellt. Hier ist Typ-S nicht die Anerkennung des Fachthemas der anderen Gruppen wichtig, sondern nur die Bewertung der Gruppe durch andere. Typ-S, so klingt es aus dem Portfolio an, scheint mehr Wert darauf zu legen, dass die Gruppenarbeit «als Gruppe» sehr gut bewertet wurde, als das fachliche Ergebnis der Gruppenarbeit. Auf persönlichen Erfolg legt sie anscheinend wenig Wert, denn sie identifiziert sich ausschliesslich mit der Gruppe, in der sie eine Publikumsrolle ohne eine Leistungsrolle (Luhmann, 1999) inne hat. Kooperativ verhält sich Typ-S auch den anderen 
Gruppen gegenüber und erkennt deren Leistungen an: "Gespannt war ich auch auf die Ergebnisse der anderen Teams».

Typ-S resümiert über das Modul als Vorfreude auf einen neuen Gruppenprozess in einem weiteren Online-Kurs. Sie wünscht als Lerneffekt, sich «kontinuierlicher und effektiver in meiner neuen Gruppe» einzubringen.

\section{Typ-F - Fachlich-Methodische Kompetenz}

Dem Typ-S, der den Gruppenprozess in den Fokus seiner Handlungen im Kurs stellt, steht als kontrastierender Fall der Kompetenztyp «fachlich-methodisch» (hier: Typ-F, weibl.) entgehen. Fachlich-methodische Kompetenzen werden bei Erpenbeck \& von Rosenstiel als die Dispositionen einer Person definiert, «bei der Lösung von sachlich-gegenständlichen Problemen geistig und physisch selbstorganisiert zu handeln, d. h. mit fachlichen und instrumentellen Kenntnissen, Fertigkeiten und Fähigkeiten kreativ Probleme zu lösen, Wissen sinnorientiert einzuordnen und zu bewerten; das schliesst Dispositionen ein, Tätigkeiten, Aufgaben und Lösungen methodisch selbstorganisiert zu gestalten, sowie die Methoden selbst kreativ weiterzuentwickeln» (ebd., 2003, S. XVI ).

typ_F_weibl

Sozialkommunikative Kompetenz\Problemlösungsfähigkeit / Akzent Aktivitätskompetenz Sozialkommunikative KompetenzlGewissenhaftigkeit / Akzent Fachkompetenz

Fach- und MethodenkompetenzlSystematisch-methodisches Vorgehen / Akzent Aktivitätskompetenz 2

Fach- und Methodenkompetenz \Sachlichkeit / Akzent Personale Kompetenz

Fach- und Methodenkompetenz Planungsverhalten / Akzent Fachkompetenz

Fach- und Methodenkompetenz \Marktkenntnisse / Akzent Fachkompetenz

Fach- und Methodenkompetenz $\backslash$ Fachübergr. Kenntnisse / Akzent Fachkompetenz

Fach- und Methodenkompetenz \Folgebewusstsein / Akzent Sozialkompetenz

Fach- und Methodenkompetenz $\backslash F$ leiß / Akzent Aktivitätskompetenz

Fach- und Methodenkompetenz $\backslash$ Fachwissen / Akzent Fachkompetenz

Aktivitäts- und HandlungskompetenzZZielorientiertes Führen / Akzent Fachkompetenz

Abb. 4: Kodeverteilungen Typ-F aus dem Code-Matrix-Browser von MAX-QDA

In der Kodeverteilung lassen sich 12 Fach- und Methodenkompetenzen finden. Auch die Aktivitäts- und Handlungskompetenz bezeichnet einen Mischtyp zur Fach- und Methodenkompetenz. Die beiden Ausprägungen der sozialkommunikativen Kompetenzen beinhalten die Problemlösungsfähigkeit und die Gewissenhaftigkeit in der Gruppe. Hier findet sich im Portfolio fast in Reinform ein «Fachmensch» (Max Weber) wieder.

Eine Vorbereitung des Moduls wird nicht von dieser Studentin im Portfolio thematisiert, so dass die Phase vor dem Kurs für die Analyse unbekannt bleibt. Der 
Fokus des Moduls liegt auf dem Kennenlernen von neuen Onlineinstrumenten, die innerhalb der Gruppe auf ihre Verwendbarkeit in Onlinekursen getestet werden. Das steht konträr zum Typ-S, bei dem der Fokus auf dem Gruppenprozess des Lerneffekts liegt. Um die Prüfung der Instrumente ordnet sich für Typ-F der Gruppenprozess als notwendiger «Weg zum Ziel». Den persönlichen Lernerfolg definiert die Studentin darin, sich «Wissen über technische Instrumente» anzueignen und «auszuprobieren» (F\Fachwissen u. Fach- und Methodenkompetenz \Fleiss).

Typ-F sieht die Herausforderung der Projektarbeit in der fachlichen Perspektive. Es geht hier darum, Onlineinstrumente zu recherchieren, sortieren, ordnen und auszuprobieren. Das Thema des Gruppenprojekts beinhaltet den Einsatz von internetgestützten Instrumenten in der Netzwerkarbeit. Typ-F integriert sich in eine Gruppe, in der auch der fachliche Aspekt im Vordergrund zu stehen scheint. Die Themenwahl gestaltet sich ans Fachthema angepasst: «Zunächst wurden dazu die zentralen Begriffe Lernziel, Lerneffekt und internetgestütztes Lernen definiert und die didaktischen Theorien des Lernens näher betrachtet» (FISystematisch-methodisches Vorgehen). Dann wurde «das gefundene Instrument nach Möglichkeit ausprobiert und die Anwendung den anderen beiden Projektteilnehmerinnen beschrieben» (F \Sachlichkeit u. Fach- und Methodenkompetenz \Planungsverhalten).

Im Gegensatz zum Typ-S, der die Herausforderung in der Projektarbeit im Zusammenspiel der Projektgruppe sieht, ist für Typ-F die Herausforderung während der Projektarbeit, "die Vielfalt der Instrumente zu ordnen und dann die einzelnen Instrumente alle auszuprobieren» (F\Planungsverhalten).

Die anderen Gruppenmitglieder erwähnt Typ-F mit keinem Wort. Lediglich wird ein Kapitel mit «Eigene Anteile an der Projektarbeit» benannt. Diese eigenen Anteile bestehen in der Übernahme der Definition der «Lernziele und Lerneffekte». Zu vermuten sei die Eingebundenheit in der Gruppe als "Rädchen im Getriebe», denn Typ-F legt hiermit die fachliche und nicht seine soziale Position der Gruppe dar. Typ-F besetzt somit in der Gruppe eine sachliche, durch Fachwissen legitimierte Position. Vor allem verantwortlich für die Definition des Fachthemas und nicht für den Erhalt eines kommunikativen Prozesses in der Gruppe.

Den «einzigen Nachteil» in einer Online-Intensivphase (Präsentation der Projektergebnisse) sieht Typ-F in der zeitlichen Belastung («bin ich vor der Präsentationsphase allerdings an meine Belastungsgrenzen gestossen»). Hier werden die «Grenzen» der Studentin sichtbar, da sie den technischen Aufwand des Projekts sehr hoch einschätzt und die zeitliche Dauer unterschätzt. Zu vermuten ist beim Typ-F ein hoher fachlicher Anspruch an die Qualität der Ergebnisse, da die Belastung so gerechtfertigt wird, dass, indem der technische Aufwand steigt, der Anspruch auf die Projektinhalte zusehends unwichtiger wird. Das untermauert das Bild des fachlichen Anspruchs eines Menschen mit hoher Fach- und Methoden- 
kompetenz, dass dieser bei "Gefährdung» seines fachlichen Inhalts an eine persönliche Belastungsgrenze stösst.

Bei der abschliessenden Präsentation der Gruppenergebnisse ist Typ-F der optimale Einsatz der recherchierten Instrumente wichtig. Die Gruppe kann als «Mittel zum Zweck» gedeutet werden, um das fachliche Ergebnis zu erreichen. Mehr an Informationen über den Gruppenprozess reflektiert Typ-F in dem Portfolio nicht. Abschliessend wird das Modul auf den fachlichen Nutzen hin bewertet, der hier positiv ausfällt: «Durch das Projekt kann ich mich in meiner Arbeit nun besser darauf fokussieren, welche Instrumente wirklich für meine Zielgruppe geeignet sind». Als sogenannte Nachbereitung in einer quasi «Post-Projektarbeit» sucht Typ-F weiter nach Instrumenten und Tools zur Onlinebetreuung im Internet, die dann auf ihre Nützlichkeit hin überprüft werden.

\section{Typ-P - Personale Kompetenz}

Der nun folgende Kompetenztyp «Personale Kompetenz» (Typ-P) kann ebenso den beiden bisher vorgestellten Typen «sozialkommunikativ» und "fachlich-methodisch» als Kontrast gegenübergestellt werden. Dieser Kompetenztyp wird laut Erpenbeck \& Rosenstiel (2003) als Dispositionen einer Person beschrieben, «reflexiv selbstorganisiert zu handeln, d. h. sich selbst einzuschätzen, produktive Einstellungen, Werthaltungen, Motive und Selbstbilder zu entwickeln, eigene Begabungen, Motivationen, Leistungsvorsätze zu entfalten und sich im Rahmen der Arbeit und ausserhalb kreativ zu entwickeln und zu lernen» (ebd., 2003, S. XVI ).

In diesem Portfolio lässt sich nach Abgleich des Schemas von Heyse \& Erpenbeck ein Student identifizieren, der über ein hohes Mass an personaler Kompetenz verfügt. Anders als bei den beiden vorangegangenen Typen sind hier die personalen Kompetenzen in einer hohen Konzentration vorhanden, die allerdings wenig für eine Gruppenarbeit nützlich sind.

Typ-P gruppiert sich das Modul samt seiner Projektgruppe um seine persönlichen Kursziele herum und reflektiert den Kurs als «Einzellerner», der von Erwartungen getragen wird, dass der gesamte Kurs auf seine Bedürfnisse zugeschnitten ist. Zur Erinnerung: Bei dem sozialkommunikativen Kompetenztyp fokussierte sich das Online-Modul auf den Gruppenprozess, bei dem fachlich-methodischen Typ auf das Fachthema. 


\begin{tabular}{|c|c|}
\hline typ_P_maennl & \\
\hline Persönliche Kompetenz\Zuverlässigkeit / Akzent Fachkompetenz & 1 \\
\hline Persönliche Kompetenz\Selbstmanagement / Akzent Aktivitätskompetenz & 2 \\
\hline Persönliche Kompetenz\Mitarbeiterförderung / Akzent Sozialkommunikative Kompetenz & 1 \\
\hline Persönliche KompetenzlLernbereitschaft / Akzent Fachkompetenz & 1 \\
\hline Persönliche KompetenzlEigenverantwortung / Akzent Personale Kompetenz & 8 \\
\hline Persönliche Kompetenz\Ganzheitliches Denken / Akzent Fachkompetenz & 1 \\
\hline Persönliche Kompetenz\Einsatzbereitschaft / Akzent Aktivitätskompetenz & 1 \\
\hline Sozialkommunikative Kompetenz\Problemlösungsfähigkeit / Akzent Aktivitätskompetenz & 1 \\
\hline Fach- und MethodenkompetenzlSyst.-method.Vorgehen / Akzent Aktivitätskompetenz & 2 \\
\hline Fach- und Methodenkompetenz\Planungsverhalten / Akzent Fachkompetenz & 1 \\
\hline Fach- und Methodenkompetenz $\backslash$ Fleiß / Akzent Aktivitätskompetenz & 1 \\
\hline Aktivitäts- und HandlungskompetenzไZielorientiertes Führen / Akzent Fachkompetenz & 2 \\
\hline Aktivitäts- und Handlungskompetenz\Initiative / Akzent Aktivitätskompetenz & 1 \\
\hline Aktivitäts- und Handlungskompetenz\Ergebnisorientiertes Handeln / Akzent Fachkompetenz & 2 \\
\hline Aktivitäts- und Handlungskompetenz\Beharrlichkeit / Akzent Fachkompetenz & 1 \\
\hline
\end{tabular}

Abb. 5: Kodeverteilungen Typ-P aus dem Code-Matrix-Browser von MAX-QDA

Auffällig ist die Verwendung des Wortes «ich» in diesem Portfolio. Viele Sätze beginnen mit «von mir pünktlich beantwortet wurden», «wählte ich entsprechend meiner geschilderten Erwartungen», «stelle ich zunächst in den Aufgabenbereich», «wurde von mir gepostet», und so weiter.

Bei Typ-P beginnt die intensive Beschäftigung mit dem Modul bereits vor dem offiziellen Kursstart. Er recherchiert intensiv die Funktionen der Online-Tools, um nicht unvorbereitet in das Modul zu gehen («Zur Vorbereitung testete ich die Funktionen von WebCT und studierte die dort angebotenen Arbeitshilfen und Materialien, um auf die Anforderungen im Modul vorbereitet zu sein»). Dies zeigt einerseits ein hohes Mass an persönliche Kompetenz der Eigenverantwortung, könnte andererseits in eine egozentrierte Sichtweise führen. Heyse \& Erpenbeck geben für jeden Kompetenztyp eine Stärken- und Schwächenzuschreibung. Der Typ-P, der hier in den Portfolios identifiziert wurde, ist in einem Gruppenprojekt als «Einzelkämpfer» zu interpretieren, der allein sein eigens Ziel vor Augen hat und mit Hilfe der Kursmitglieder auch dieses durchsetzt und -führt.

Ziel von Typ-P ist es, seinen «Kurs um sein zentrales Berufsthema zu organisieren». Somit wird das Onlinemodul zu einem passgenauen, auf ihn zugeschnittenen Lehrgang. Vorab stehen eine sorgfältige Planung und eine Auslotung der technischen und fachlichen Möglichkeiten, ob es auf seine Erwartungen passt. Die Einschätzung anderer über den Lehrgang ist von grosser Bedeutung. Hierzu wird Beratungsleistung über das Modul in Anspruch genommen («mich eingehend beraten 
liess») und dann sorgfältig der Nutzen des Lehrgangs bezüglich seiner beruflichen Ziele und Wünsche abgewogen («darüberhinaus befragte ich zwei mir durch Herrn $X$ vermittelte Studierende per E-Mail über ihre Erfahrungen»). Weiterhin sieht TypP dieses Onlinemodul als Möglichkeit, das «Studium zu testen».

Gegenüber Problemlösungsstrategien verhält sich Typ-P, ebenso wie Typ-S in dieser Studie, passiv. Nur dass er sich nicht wie Typ-S abwartend verhält, sondern bei Problemen, insbesondere technischer Art, sich «beschwert», aber nichts zur konkreten Problemlösung beiträgt. Bei technischen Problemen erwartet er eine sofortige Lösung von der Universität, ohne zu überprüfen, ob die technischen Schwierigkeit mit seiner Internetverbindung aus dem Ausland zusammenhängen könnten. Kommunikationsmissverständnisse werden von Typ-P auf den Dozenten geschoben («aufgrund von Kommunikationsproblemen mit dem Dozenten hatten leider nicht alle Teilnehmer eine Powerpointpräsentation vorbereitet»).

Wo Typ-S «Berührungsängste» mit dem fremden Online-Tool zeigt, äussert Typ$\mathrm{P}$ «Bedenken», dass er aufgrund eventuell auftretender technischer Fehler nicht «optimal starten» könnte. Wie im nächsten Kapitel noch zu zeigen sein wird, nutzt der Kompetenztyp-A (aktivitäts- und handlungsorientiert) technische Probleme als Herausforderung, um den richtigen Umgang mit neuen technischen Tools zu erlernen.

Bemerkenswert ist dieses Vorgehen insofern, als die Reflexionen in den Portfolios aus dem gleichen Online-Modul stammen, das gleiche Modul somit unter verschiedenen Perspektiven aus dem Blickwinkel verschiedener Kompetenztypen betrachtet wird.

Die Einstellung von Typ-P zur Online-Projektarbeit, zur Wahl des Themas und zur Gruppe ist von dem Gedanken durchzogen, sein Projekt zu verwirklichen. Typ-P wählt ein Thema mit direktem Bezug zu seiner Berufswelt, dem sich andere anschliessen (dürfen). Dazu schreibt er folgendes: «Die Projektskizze stellte ich zunächst in den Aufgabenbereich von WebCT und nicht in das allgemeine Forum und musste damit das erste Mal Tribut als Lernender zahlen. Nachdem dieser Fehler korrigiert war und die Skizzen durch den Dozenten ausführlich kommentiert waren, fanden sich mit $X$ und $Y$ zwei Teilnehmer, die sich dem Projekt anschlossen». Er schlägt das Thema nicht in einem demokratischen Diskurs vor, sondern veröffentlicht vorab das Thema allein im Forum und lässt es vom Dozenten ausführlich kommentieren. Dann wählt er «seine» Gruppenmitglieder nach fachlichen Kenntnissen und «Effizienz» für ihn aus, die sich dem Thema anschliessen, welches er nun federführend organisiert und seine Gruppenmitglieder so zu Randfiguren degradiert. Eine «perfekt funktionierende» personale Kompetenz, die zeigt, dass er eine hohe Eigenverantwortung besitzt und "gut für sich sorgt».

Seine Position in der Gruppe wird zur Position des «Vorreiters» und «Bewerters», bei dem es kennzeichnend hier für Typ-P ist, dass die Gruppenarbeit nach seinen Regeln reibungslos funktioniert ( «...dass die von mir vorgelegte Projektidee 
Basis der weiteren Zusammenarbeit sein sollte»). Unerwartete Zwischenfälle, wie eine plötzlich auftretende Problemsituation eines Gruppenmitglieds, gefährden das Fortkommen der Gruppenarbeit. Bei der Präsentation der Ergebnisse kam es «im Falle von Frau $X$ für einige Tage zu einer ernsten privaten Problemsituation». Einzelne konkrete sozialkommunikative Kompetenzen können hier nicht zur Problemlösung und als Empathie für Frau X identifiziert werden. Typ-P berichtet, dass durch das schnelle Eingreifen der Gruppe die Präsentation seiner Projektidee nicht blockiert wurde.

Im Unterschied zu dem sozialkommunikativen Typ-S, zeigt sich hier deutlich der Fokus auf die eigene Projektidee, wohingegen dem sozialkommunikativen Kompetenztyp in einer Problemsituation das Wohlergehen der Gruppenkollegin wichtig ist. Die Regel «Einer für Alle - und Alle für Einen» des sozialkommunikativen Typs scheint beim Typ mit ausgeprägter personaler Kompetenz zum Prinzip «Alle für Mich» zu werden. Dagegen könnte beim fachlich-methodischen Kompetenztyp eher von einem «Alle für das gemeinsame Thema » gesprochen werden.

Stärken zeigt Typ-P mittels der Kompetenzen «Beharrlichkeit» und «Fleiss». So listet er detailgetreu in seiner Reflexion die Posten seiner verrichteten Aufgaben in der Gruppe auf («folgende Arbeiten wurden von mir erledigt»). Das beinhaltet Arten von Aufgaben, die die Basis für weitere Aufgaben der Gruppenmitglieder bilden, z. B. Literaturrecherche, Errichtung einer Internetplattform. Implizit klingt an, dass die Gruppe ohne seine «Basisarbeit» nicht weiterkommen könnte, indem er beispielsweise anführt: «Aus dieser Tabelle wurden durch mich auch alle Diagramme für die Präsentation generiert.»

Das Fazit von Typ-P nach dem Kurs läuft auf rein persönliche Interessen hin: «Gut gefallen hat mir auch, dass ich meinen beruflichen Hintergrund einbringen konnte und die Freiheit hatte, als Teil einer sehr engagierten Projektgruppe, an einer praxisbezogenen Fragestellung zu arbeiten. Unsere Ergebnisse sind teilweise schon in ein konkretes Projekt eingeflossen.» Unthematisiert bleibt, wie die Gruppenmitglieder dieses «Gruppenergebnis», bei dem sie als «Nebenakteure» fungieren, in ihre eigene berufliche Tätigkeit integrieren können.

Nach Ende der Projektphase schliesst Typ-P noch mit einem «letzten Wort» den Kurs ab, indem er ein Feedback mit u. a. Verbesserungsvorschlägen im Forum an den Tutor verfasst.

Auch bei diesem gefundenen Kompetenztyp-P erweist sich das Raster von Heyse \& Erpenbeck als geeignet, hier einen eindeutigen Kompetenztyp zu identifizieren. Inhaltlich stimmen diese zugeschriebenen Kompetenzen von Heyse \& Erpenbeck auch mit dem Verhalten des Studenten überein. Würde man zum Beispiel den Text mit einer textanalytischen Methode untersuchen, so würde man hier auch einen Akteur herausfinden, der aus einer persönlichen Nutzen-Kostenkalkulation heraus agiert. 


\section{Typ-A - Aktivitäts- und Handlungskompetenz}

Ähnlich dem Typ-P im Kompetenzbereich Fleiss und Engagement verfügt der Aktivitäts- und Handlungskompetenztyp (Typ-A) über eine hohe Motivation. Die Schaffenskraft ist bei beiden Kompetenztypen hoch ausgeprägt, wenngleich die Intentionen, die das Handeln motivieren, bei Typ-A aus Innovationsfreudigkeit und Begeisterung am Thema herführen und nicht aus persönlicher Zielerreichung.

Erpenbeck \& Rosenstiel (2003) bezeichnen die Aktivitäts- und Handlungskompetenz als Dispositionen einer Person, «aktiv und gesamtheitlich selbstorganisiert zu handeln und dieses Handeln auf die Umsetzung von Absichten, Vorhaben und Plänen zu richten - entweder für sich selbst oder auch für andere und mit anderen, im Team, im Unternehmen, in der Organisation. Diese Dispositionen erfassen damit das Vermögen, die eigenen Emotionen, Motivationen, Fähigkeiten und Erfahrungen und alle anderen Kompetenzen - personale, fachlich-methodische und sozialkommunikative - in die eigenen Willensantriebe zu integrieren und Handlungen erfolgreich zu realisieren» (ebd., 2003, S. XVI).

typ_A_weibl

Sozialkommunikative KompetenzlTeamfähigkeit / Akzent Personale Kompetenz Sozialkommunikative Kompetenz\Problemlösungsfähigkeit / Akzent Aktivitätskompetenz

Fach- und MethodenkompetenzlSystematisch-methodisches Vorgehen / Akzent Aktivitätskompetenz Fach- und Methodenkompetenz Projektmanagement / Akzent Sozialkompetenz Fach- und Methodenkompetenz \Folgebewusstsein / Akzent Sozialkompetenz

Aktivitäts- und HandlungskompetenzlSoziales Engagement / Akzent Sozialkompetenz Aktivitäts- und Handlungskompetenz〉Optimismus / Akzent Sozialkompetenz Aktivitäts- und Handlungskompetenz\Ausführungsbereitschaft / Akzent Aktivitätskompetenz Aktivitäts- und HandlungskompetenzInitiative / Akzent Aktivitätskompetenz Aktivitäts- und HandlungskompetenzInnovationsfreudigkeit / Akzent Personale Kompetenz Aktivitäts- und HandlungskompetenzlGestaltungswille / Akzent Personale Kompetenz Aktivitäts- und HandlungskompetenzlErgebnisorientiertes Handeln / Akzent Fachkompetenz Aktivitäts- und Handlungskompetenz/Beharrlichkeit / Akzent Fachkompetenz

Abb. 6: Kodeverteilungen Typ-A aus dem Code-Matrix-Browser von MAX-QDA

Die Reflexion von Typ-A beginnt mit einem englischen Zitat über die vielfältigen Möglichkeiten von Online-Tools zur Vernetzung der Lernenden. In der technischen Innovation und Weiterentwicklung sieht Typ-A die Herausforderungen des OnlineLernens und auch die persönliche Herausforderung, dieses Modul zu belegen.

Typ-A benennt im ersten Abschnitt im Portfolio die Motivation für die Aufnahme der Weiterbildung («getrieben vom Praxisbezug»). Die Studentin beschreibt das erste Kapitel als «Motivation bezüglich des Projektthemas», was auf ihre Intention einer motivationalen Zielrichtung zur Aufnahme des Moduls führt. Zum Vergleich: Typ-S (sozialkommunikativ) beginnt das Portfolio mit «Reflexion der Gruppenpha- 
se», Typ-P (personale Kompetenz) berichtet im ersten Abschnitt von seinen persönlichen Zielen im Kurs und Typ-F (fachlich-methodische Kompetenz) leitet den Einführungstext mit der fachlich sachlichen Beschreibung des Projektziels ein.

Parallel zu dem untersuchten Onlinemodul ist die Studentin Typ-A in die Betreuung und Organisation eines weiteren Fernstudiums eingebunden. Typ-A zeichnet sich über ihre Reflexionen im Text durch eine hohe Motivation und Interesse am Studiengang aus. Technische Probleme beschreibt Typ-A im Portfolio als «Herausforderungen», um den Umgang mit technischen Online-Tools zu lernen. Auffällig sind hier die positiv geprägten Aussagen im Text. Typ-A spricht von Motivation, Interesse, Herausforderungen, Mehrwert, Gewinne, Prozess, usw.

Auch bei der Bearbeitung der Gruppenarbeit wird dieses Prozessdenken deutlich. Typ-A resümiert: «Hatten wir anfangs noch das Ziel, einen qualitätssichernden Massnahmenkatalog zu erstellen, so entwickelte sich im Verlauf des Projekts die Erkenntnis, dass dies aufgrund der Einzigartigkeit jeder Organisation kein gewinnbringendes Vorhaben ist, da die jeweiligen qualitätssichernden Massnahmen für jede Institution unterschiedlich sind».

Die Position in der Projektgruppe ist aktiv teilnehmend und kollegial. Es herrscht eine Gleichberechtigung in der Gruppe, die sich nach Kompetenzen der einzelnen Gruppenmitglieder gebildet hat und auch nach den jeweiligen Fähigkeiten die Aufgabenverteilung vornimmt («schnell waren die Prioritäten und die Kompetenzen der einzelnen Gruppenmitglieder klar definiert»). Typ-A bezeichnet die Gruppe als «Dreamteam».

Die Studentin Typ-A widmet einen langen Absatz ihres Portfolios ihrem parallelen Fernstudium und der Tätigkeitbeschreibung als Semesterbetreuerin der gleichen Hochschule. Viele Abläufe in ihrem Tätigkeitsbereich sind nicht definiert und aufgrund ihrer «Internetaffinität» wird die Studentin im Laufe der Zeit «nebenbei zur E-Learning-Beauftragten der gesamten Hochschule». Trotz Doppelbelastung ihrer Tätigkeit und Absolvierung des Online-Moduls scheint diese Situation Typ-A noch «aktiver» und motivierter werden zu lassen, denn «diese Situation steigerte ihr Interesse», sich noch intensiver mit der Thematik auseinanderzusetzen. Zum Kontrast das Beispiel der «Belastungsgrenze» von Typ-F: Typ-F kam an der «Belastungsgrenze» unter Zeitdruck, da hier die Qualität des Fachthemas nicht gewährleistet werden konnte, wohingegen Typ-A unter «Zeitdruck» ihr Pensum noch steigert und das Interesse am Projekt wachsen lässt.

Die Onlinepräsentation sieht Typ-A als Herausforderung, die «technische Umsetzung der Internetpräsentation zu bewältigen». Gleichzeitig entdeckt sie auch hier den Vorteil, dass «ihr damit Reisen erspart blieben».

Das Ziel der Weiterbildung meint Typ-A vollständig erreicht zu haben und sieht einen «Gewinn für die Praxis», wie durch eine Kapitelüberschrift verdeutlicht: «Tatsächlich kann ich die gewonnenen Ergebnisse nun zu hundert Prozent in meine berufliche Praxis übertragen.» 
Für Typ-A in dieser Untersuchung sind bei den sozialkommunikativen Kompetenzen die Bereiche Teamfähigkeit und Problemlösungsfähigkeit mit einem hohen Aktivitätsakzent zu erkennen. Bei der Fach- und Methodenkompetenz fällt das systematisch-methodische Vorgehen, die Fähigkeit zum Projektmanagement und das Folgebewusstsein auf, welches eine Mischform Fach- und Methodenkompetenz / Sozialkompetenz bedeutet.

\section{Zusammenfassung und Fazit}

Abschliessend kann resümiert werden, dass sich das Kompetenzraster von Heyse \& Erpenbeck als geeignetes Instrument zeigt, Kompetenzen von Studierenden in ihren Portfolios sichtbar zu machen und somit die vorhandenen Kompetenzen der Studierenden zu identifizieren. Ferner kann das Raster dazu beitragen, anhand der Reflexionen der Studierenden auf die Art der Kompetenzen zu schliessen, die in Lernprozessen erworben wurden und zu erkennen, in welchen Lernsituationen oder bei welchen auftretenden Problemen die Studierenden welche Kompetenzen einsetzen oder wo die Grenzen der Studierenden liegen.

Eine Erklärung für die Ursachen und die Entstehung der gefundenen Kompetenzen kann allerdings diese Textanalyse nicht leisten. Es ist somit nicht feststellbar, ob und welche der identifizierten Kompetenzen vor der Belegung des Moduls bereits vorhanden waren bzw. während des Lernprozesses erworben oder modifiziert wurden. Eine kausal auf das Modul rückführbare Kompetenzerweiterung oder -veränderung lässt sich über die Textanalyse nicht ermitteln. Interessant wäre anhand von Längsschnittstudien zu verfolgen, ob sich die identifizierten Typologien im Studienverlauf infolge der sozialen Konstruktion des Instruktionsdesigns des Studiums verändert.

Die Textanalyse anhand des Kompetenzrasters ermöglicht aber, Kompetenzen der Studierenden weitaus differenzierter zu identifizieren, als dies mit anderen Prüfungsformen möglich wäre. Auch wenn sich nicht ableiten lässt, warum die Studierenden ausgerechnet über diese und nicht andere Kompetenzen verfügen und ursächliche Gründe für das Verhalten der Studierenden während der Projektphase oder der Reflexionsphase aus dem Text, der in Form eines Protokolls oder Arbeitsberichtes abgefasst wurde, nicht erschliessbar sind, ermöglicht dieses Verfahren doch ein kriteriengeleitetes Feedback.

Voraussetzung hierfür ist, dass kompetenzbasierte Studiengänge auf anerkannten Kompetenzmodellen basieren, die dazu beitragen, dass ein Portfolio nicht nur eine Ansammlung von Artefakten ist, sondern eine systematische Darstellung erworbener Kompetenzen, die sich einer Einschätzung durch Dritte erschliessen. Das in diesem Beitrag verwendete Modell scheint hierfür gut geeignet zu sein. Es muss jedoch für die Studierenden transparent gemacht werden, so dass sie sich explizit darauf beziehen können. Die meisten Lehrenden oder gar Arbeitgeber werden sich nicht die Mühe einer qualitativen Textanalyse machen. 


\section{$5 \quad$ Literatur}

Baumgartner, Peter; Himpsl, Klaus \& Zauchner, Sabine (2009). Einsatz von E-Portfolios an (österreichischen) Hochschulen. Krems: Donau Universität Krems, Department für Interaktive Medien und Bildungstechnologien.

Brüsemeister, Thomas (1999). Qualitative Sozialforschung. Ein Überblick. Studienbrief der FernUniversität Hagen Fachbereich Erziehungs-, Sozial- und Geisteswissenschaften, Hagen.

Connell, Michael W.; Sheridan, Kimberly \& Gardner, Howard. (2003). On abilities and domains. In Robert J. Sternberg \& Elena Grigorenko (Hrsg.), The psychology of abilities, competencies and expertise. Cambridge: Cambridge University Press. S. 126-155.

Erpenbeck, John \& Heyse, Volker (2007). Die Kompetenzbiographie - Wege der Kompetenzentwicklung. Münster: Waxmann.

Erpenbeck, John \& Heyse, Volker (1999). Die Kompetenzbiographie - Strategien der Kompetenzentwicklung durch selbstorganisiertes Lernen und multimediale Kommunikation. Münster: Waxmann.

Erpenbeck, John \& von Rosenstiel, Lutz (Hrsg.). (2003). Handbuch Kompetenzmessung - Erkennen, verstehen und bewerten von Kompetenzen in der betrieblichen, pädagogischen und psychologischen Praxis. Stuttgart: SchäfferPoeschel.

Flick, Uwe (1996): Qualitative Sozialforschung: Eine Einführung. Reinbek bei Hamburg: Rowohlt-Taschenbuch-Verlag.

Heyse, Volker \& Erpenbeck, John (2004). Kompetenztraining - 64 Informationsund Trainingsprogramme. Stuttgart: Schäffer-Poeschel.

Knowles, Malcolm Shepherd (1975). Self-directed learning: A guide for learners and teachers. Englewood Cliffs, NJ: Cambridge Adult Education.

Kuckartz, Udo (2007). Einführung in die computergestützte Analyse qualitativer Daten. Wiesbaden: VS Verl. für Sozialwissenschaft.

Luhmann, Niklas (1999). Die Wirtschaft der Gesellschaft. Frankfurt am Main: Suhrkamp.

Reinmann, Gabi (2007). Arbeitsbericht 16: Bologna in Zeiten des Web 2.0 - Assessment als Gestaltungsfaktor. Augsburg: Universität Augsburg, Institut für Medien und Bildungstechnologie.

Sauter, A. M. \& Sauter, W. (2002). Blended Learning - Effiziente Integration von ELearning und Präsenztraining. Neuwied, Kriftel: Luchterhand.

Stratmann, J.; Preussler, A. \& Kerres, M. (2009). Lernerfolg und Kompetenz: Didaktische Potentiale der Portfolio-Methode im Hochschulstudium. Zeitschrift für Hochschulentwicklung, 4(1), S. 90-103.

Strauss, Anselm \& Corbin, Juliet (1996). Grounded Theory: Grundlagen qualitativer Sozialforschung. Weinheim: Beltz. 
Schnell, Rainer.; Hill, Paul \& Esser, Elke (1992). Methoden der empirischen Sozialforschung. München: Oldenbourg.

Walti, Christine (2004). Implementing web-based portfolios and learning journals. In Jane E. Brindley, Christine Walti \& Olaf Zawacki-Richter (Hrsg.), Learner support in open, online and distance learning environments. Oldenburg: Bibliotheks- und Informationssystem der Universität Oldenburg. S. 157-168.

Weinert, Franz E. (1996). Lerntheorien und Instruktionsmodelle. In Franz E. Weinert (Hrsg.), Enzyklopädie der Psychologie, Psychologie des Lernens und der Instruktion. Göttingen: Hogrefe. S. 1-48.

Weinert, Franz E. (2001). Concept of competence: A conceptual clarification. In Dominique Simone Rychen \& Laura Hersh Salganik (Hrsg.), Defining and selecting key competencies. Göttingen: Hogrefe \& Huber Publishers. S. 45-65.

Zawacki-Richter, Olaf; Hasebrook, Joachim, \& Muckel, Petra (2009). Web 2.0 basierte Ansätze zur Unterstützung innovierenden Handelns in Unternehmen. In Kreativität und Innovationskompetenz im digitalen Netz: E-creativity und EInnovation. Salzburg: Salzburg Research Verlag.

Zawacki-Richter, Olaf \& Hasebrook, Joachim (2005). Soft Skills online? Lernziel interkulturelle Kompetenz. In D. Tavangarian \& K. Nölting (Hrsg.), Auf zu neuen Ufern! E-Learning heute und morgen. Münster: Waxmann. S. 17-26. 